\title{
Inducing Chaos in Electronic Circuits by Resonant Perturbations
}

\author{
Anil Kandangath, Satish Krishnamoorthy, Ying-Cheng Lai, and John A. Gaudet, Member, IEEE
}

\begin{abstract}
We propose a scheme to induce chaotic attractors in electronic circuits. The applications that we are interested in stipulate the following three constraints: 1) the circuit operates in a stable periodic regime far away from chaotic behavior; 2) no parameters or state variables of the circuit are directly accessible to adjustment and 3) the circuit equations are unknown and the only available information is a time series (or a signal) measured from the circuit. Under these conditions, a viable approach to chaos induction is to use external excitations such as a microwave signal, assuming that a proper coupling mechanism exists which allows the circuit to be perturbed by the excitation. The question we address in this paper is how to choose the waveform of the excitation to ensure that sustained chaos (chaotic attractor) can be generated in the circuit. We show that weak resonant perturbations with time-varying frequency and phase are generally able to drive the circuit into a hierarchy of nonlinear resonant states and eventually into chaos. We develop a theory to explain this phenomenon, provide numerical support, and demonstrate the feasibility of the method by laboratory experiments. In particular, our experimental system consists of a Duffing-type of nonlinear electronic oscillator driven by a phase-locked loop (PLL) circuit. The PLL can track the frequency and phase evolution of the target Duffing circuit and deliver resonant perturbations to generate robust chaotic attractors.
\end{abstract}

Index Terms-Duffing oscillator, inducing chaos, phase-locked loop (PLL), resonant perturbations.

\section{INTRODUCTION}

$\mathbf{M}$ ANY modern devices rely on sophisticated electronic circuits. An important class of these devices is electronic tracking and guidance systems. To accomplish its intended mission, a hostile electronic tracking and guidance system operates in a parameter regime where their performance can be characterized as stable or regular. If one regards the device as a dynamical system, it is required that the state variables of the system behave in a regular fashion. This is conceivable because the electronic circuits in the system, when in operation, are indeed dynamical systems that evolve state variables such as voltages and currents continuously in time. Inducing chaos in the circuits is likely to cause the system to fail in its intended mission.

There have been many previous works on inducing or maintaining chaos in nonlinear systems. These can be categorized

Manuscript received June 16, 2005; revised September 30, 2006. This work was supported by AFOSR under Grant. F49620-03-1-0290 and Grant FA9550-06-1-0024. This paper was recommended by Associate Editor Y. Nishio.

A. Kandangath, S. Krishnamoorthy, and Y.-C. Lai are with Department of Electrical Engineering, Arizona State University, Tempe, AZ 85287 USA (e-mail: anil.kumar; satishk; Ying-Cheng.Lai@asu.edu).

J. A. Gaudet is with Air Force Research Laboratory, AFRL/DEHE, Kirtland AFB, NM 87117 USA.

Digital Object Identifier 10.1109/TCSI.2007.893510 into three classes: 1) inducing chaos by random noise [1]-[11]; 2) converting transient chaos into sustained chaos by small perturbations - the problem of maintaining chaos [12]-[17] and 3) inducing chaos by resonant perturbations [18]-[23]. In the first class, the main question concerns how chaos can arise under the influence of random noise. The pioneering work of Crutchfield et al. [1], [2] established that, in the common route to chaos via period-doubling bifurcations, noise tends to smooth out the transition and induce chaos in parameter regimes where there is no chaos otherwise. The observability and scaling of fractal structures near the transition to chaos in random maps were addressed in [6], [7]. Features of transition to chaos in noisy dynamical systems, such as intermittency and the smoothness of the Lyapunov exponents, were also found in the transition from strange nonchaotic to strange chaotic attractors in quasi-periodically driven systems [24] and in the bifurcation to chaos with multiple positive Lyapunov exponents in relatively high-dimensional systems [25]-[27]. More recently, the mechanism for transition to chaos in continuous-time dynamical systems was investigated [11] where it was found that nonhyperbolicity plays a fundamental role in shaping the transition. The second class of problems deals with systems in parameter regimes where there are nonattracting chaotic sets that physically lead to transient chaos. That is, under its own evolution, from a random initial condition the system behaves chaotically only for a finite amount time before settling into a nonchaotic attractor. Since sustained chaos is believed to be beneficial in circumstances such as biological applications [13], [14], [28], it is desirable that chaos be maintained, which can indeed be achieved by applying small perturbations to an available parameter or state variable of the system [12], [16], [17]. For the third class, previous works demonstrated that for simple nonlinear oscillators such as the Duffing system, resonant perturbations can be used to drive the system in and out of chaotic motion [18]-[23], provided that the system equations are known so that the external excitation can be designed accordingly.

The problem of inducing chaos that we wish to address in this paper is significantly more challenging than those investigated previously. In particular, we are interested in applications where the following three constraints naturally arise: 1) The electronic circuit to be defeated operates in stable state that is far away from any chaotic dynamics; 2) the internal structure and parameters of the circuit cannot be modified, i.e., no parameters or state variables of the circuit are directly accessible to adjustment and 3) the system equations are unknown and only a measured signal (time series) from the system is available. In previous works, however, not all these three requirements were assumed. For instance, in the work of maintaining chaos [14], [17], [18], although system equations were unknown, the 
system was in a transiently chaotic regime and a system parameter or dynamical variable was externally accessible for continuous adjustment. In the existing resonant-perturbation approach [18]-[23], knowledge of system equations was assumed. For our problem of disturbing a target electronic circuit under the three constraints, a viable way is to radiate the circuit with external wave, e.g., microwave. Inducing chaos to interrupt the normal operation of the circuit may be regarded as advantageous because the absorbed energy required may be much less than that needed to simply "overpower" the same electronics by using large-amplitude excitations. In addition, it is generally desirable to use only small excitations to induce chaos, as it may be difficult to generate large-amplitude waves in some realistic applications.

We have developed a general approach to inducing chaos in nonlinear circuits by making use of the principle of phase-locking and resonance, without knowledge of system equations. In this paper, we shall present a theory and demonstrate the feasibility of this approach numerically and experimentally. The idea is to apply judiciously chosen perturbations to drive a target circuit into higher and higher resonant states. The perturbations can be a sinusoidal signal with time-varying frequency and phase, and how they vary is determined by a real-time measured signal from the circuit. Our goal is to control the perturbing field so as to maximize its effect on the circuit, thereby driving it as far away from its equilibrium as possible, eventually generating chaotic dynamics. To explain our method, we start with a simple one-degree-of-freedom Hamiltonian (conservative) system and argue that small-amplitude, sinusoidal excitations whose frequency and phase match approximately those of the measured signal from the system can drive it gradually into higher and higher energetic states and eventually into chaos. The key role played by the resonant perturbation is then to continuously feed energy into the system to induce instability. We address the issue of the time required to induce chaos and obtain a simple scaling relation between this time and the amplitude of the resonant sinusoidal excitation. We then consider the effect of dissipation and demonstrate that, insofar as the average rate of increase of energy in the system is positive, resonant perturbations can drive the system into chaos, whereas the time required satisfies the same scaling law as in the case of Hamiltonian system. For experimental implementation, we use a prototype of nonlinear electronic circuit, the Duffing circuit, and show that resonant perturbations from a phase-locked loop (PLL) circuit can readily drive the target circuit into chaos. Here, the role of PLL circuit is to continuously track the instantaneous frequency and phase of the target circuit and at the same time, deliver proper resonant perturbations. We shall demonstrate that excitation of amplitude from the PLL of about $10 \%$ of the maximum circuit voltage oscillation can induce robust chaotic motion in the circuit. (Here "robust" means that when a system parameter is changed, such as the driving frequency, there are no periodic windows [29] amid the induced chaotic attractors.) To demonstrate the power of the resonant-perturbation approach, we will also show that in the same parameter regime, random perturbations of much larger amplitude are unable to induce chaos. A brief account of part of the work has been published recently [30].
In Section II, we construct a simple toy model to enable an understanding of our general method of inducing chaos. The focus of this paper is experimental demonstration of the method using a PLL circuit [Section III]. A discussion is presented in Section IV.

\section{General PRinciple AND Methodology}

The general mathematical principle that enables time-dependent perturbations to cause a stable dynamical system to become chaotic is as follows. Consider a dynamical system described by a set of $N$ autonomous ordinary differential equations

$$
\frac{d \mathbf{x}}{d t}=\mathbf{f}(\mathbf{x})
$$

where $\mathbf{x} \in \mathbf{R}^{N}$ is an $N$-dimensional vector with components $\left(x_{1}, x_{2}, \ldots, x_{N}\right)$, and the velocity field $\mathbf{f}(\mathbf{x})$ is a nonlinear function. The state equations describing most typical electronic circuits can be written in the above form. Now imagine that an external excitation is applied so that (1) becomes

$$
\frac{d \mathbf{x}}{d t}=\mathbf{F}[\mathbf{x}, g(t)]
$$

where now the velocity field contains an explicit time dependence due to the time-varying excitation $g(t)$ and, hence, it is nonautonomous. Equation (2), however, can be converted into an autonomous system simply by introducing a new $(N+1)$-dimensional variable $\mathbf{y}(\mathbf{t})=[\mathbf{x}(t), g(t)]$, where $y_{N+1}=g(t)$. The dynamical equation for $\mathbf{y}$ can be written as

$$
\begin{aligned}
\frac{d y_{i}}{d t} & =F_{i}\left(y_{1}, y_{2}, \ldots, y_{N}, y_{N+1}\right), \quad i=1, \ldots, N \\
\frac{d y_{N+1}}{d t} & =\frac{d g}{d t} .
\end{aligned}
$$

The system governing the motion of the new variable $y$ has a phase-space dimension that is one higher than that of the original equation in $\mathbf{x}$. An increase in the phase-space dimension indicates a possible increase in the complexity of the system and, hence, chaos is more likely to occur in the new system. In principle, it is thus possible to induce chaos in a nonchaotic system by using external time-dependent excitations.

To explain our approach of resonant perturbation without requiring system equations, we imagine a simple, linear, conservative (Hamiltonian) system: a harmonic oscillator that is a textbook example in classical mechanics. In this system, the natural oscillating frequency $\omega$ does not depend on the energy. As a consequence, we can induce an arbitrarily large disturbance in this system by applying an arbitrarily small perturbing force at the fixed resonant frequency $\omega$. It is important to note that the resonant frequency is the same for all energies, because the natural frequency is constant. This convenient feature is, however, restricted to linear systems. For nonlinear systems, the system's natural frequency does depend on the energy, and using a perturbing field with a fixed frequency in general cannot generate resonance. Our basic idea is then to change the frequency of the 
perturbing field so as to "follow" the natural frequency of the system as the energy changes due to perturbation.

Since our system has only one degree of freedom, in the absence of external perturbations, it exhibits simple stable motion and fundamentally prohibits any chaotic motion. Demonstrating that chaos can be induced in such a stable system by small resonant perturbations illustrates the power of our method. The dynamics of the unperturbed Hamiltonian system is described by

$$
\frac{d^{2} x}{d t^{2}}=-\frac{d V(x)}{d x}
$$

where $V(x)$ is a one-dimensional potential function. For a harmonic oscillator, $V(x)$ is a quadratic function. In general, $V(x)$ can be any differentiable function. We assume, however, that $V$ has a minimum and a maximum. Although our method works for any potential satisfying these constraints, for concreteness we will focus on the pendulum potential given by

$$
V(x)=-\cos (x)
$$

The maxima at $x= \pm \pi$ define hyperbolic orbits at energy $E=1$. The hyperbolic orbits separate regions of confined and non-confined motion. Widespread chaos arises in the vicinity of the hyperbolic orbits, for arbitrarily small perturbations [31], [32]. The oscillating frequency of the unperturbed system is a function of the energy: $\omega=\omega(E)$, where $\omega$ is defined within the region of the confined motion, $-1 \leq E \leq 1$. The frequency at the minimum is $\omega(-1)=1$, and it decreases toward 0 as $E$ approaches 1, because it takes an infinite amount of time for the hyperbolic orbits to go from one maximum to the other, say from $x=+\pi$ to $x=-\pi$. We stress that this last feature is not particular of the pendulum potential, but it is true of all hyperbolic orbits: they have an infinitely long period. As the energy for a hyperbolic orbit is approached, the period diverges, and the frequency goes to zero. This is important for our method.

Consider now that the system is set up with an initial energy $E_{0}<1$. In the absence of perturbations, it will keep oscillating with this constant energy. Our goal is to apply a small perturbation so that the energy is increased toward $E_{\max }$, where the homoclinic orbits lie, around which there is sustained chaos. If we just apply a perturbation with a fixed frequency equal to the initial natural frequency $\nu_{0}=\omega\left(E_{0}\right)$, the system will rapidly fall out of resonance, as we explained. The result is that the energy $E$ oscillates around $E_{0}$, with an amplitude that decreases with the strength of the perturbation. This is shown in Fig. 1, where we use (4) with an added constant sinusoidal term $F \sin \left(\nu_{0} t+\phi_{0}\right)$, and the energy is plotted as a function of the number of oscillations of the external excitation. Since we are interested in weak perturbations (small $F$ ), it is clear that we will not be able to reach $E_{\max }$ in this way.

The key observation is that the system's natural frequency changes with the energy. We must therefore change the frequency of the perturbation so that it always matches the natural frequency, thus ensuring that the resonant condition be satisfied at all times. The frequency of the external excitation thus changes with time, and we write $\nu(t)$. The form of $\nu(t)$ cannot be be written down explicitly, because it is adjusted in response

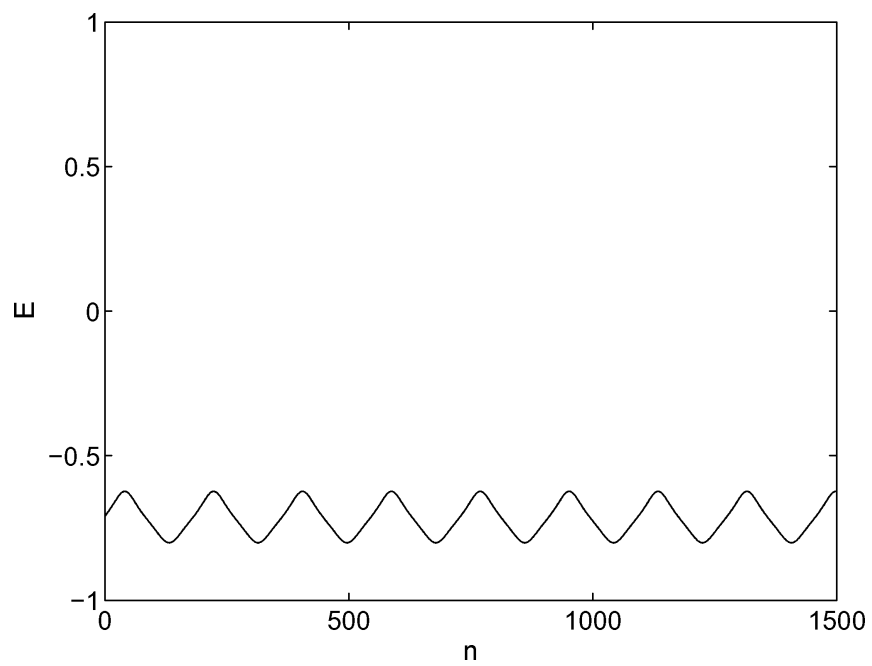

Fig. 1. Energy of the oscillator as a function of the number of oscillation periods for excitation at constant frequency, at resonance with the initial frequency. The initial conditions are $x(0)=0.5, \dot{x}(0)=0$, with a corresponding energy of $E \approx-0.88$, and the amplitude of the excitation is $F=10^{-3}$.

to the time variation of the natural frequency of the system. The equations of motion of the perturbed system can be written as

$$
\frac{d^{2} x}{d t^{2}}=-\frac{d V(x)}{d x}+F \sin [\nu(t) t+\phi(t)]
$$

where $\phi(t)$ is a time-dependent phase (to be discussed shortly). Although for the particular case of the potential (5), the frequency as a function of the energy can be expressed in terms of the elliptical functions [32], we want to keep our method as generic as possible, and so we assume such dependency is not known. In fact, we do not assume any knowledge of the potential, other than the fact that it has a minimum (with a region of confined, oscillating motion) and a hyperbolic orbit. In other words, we only require that the underlying system be oscillatory. Therefore, a practical way to determine the natural frequency of the system at a given time is through the observed dynamics, for instance, through the observation of the dynamical variable $x(t)$. We cannot measure the period directly from the dynamics, since the forcing term in (6) makes the motion aperiodic. However, since the perturbation is small $(F \ll 1)$, at any given time the motion is almost periodic, meaning that the energy changes only very slowly with time. Typically, the system oscillates many times with only a small change in $E$ and, hence, the resonant frequency changes very little as well. Using this fact, we define $\nu$ for a given time $t$ as the average over the past $\Delta n$ oscillations, where $\Delta n$ is small enough so that the energy does not change appreciably in the corresponding time interval. In this way $\nu$ is defined purely in terms of the observed quantities of the system, and any previous knowledge of the potential and/or the equations of motion are not assumed. The only requirement is that the average oscillating frequency of the system as a function of time be measured. In principle, the forcing term in (6) involves a time delay because the forcing is equivalent to a memory term. In our simulations, we consider only one oscillation in the past. The results we present seem to be independent of $\Delta n$, to within the constraint mentioned. 

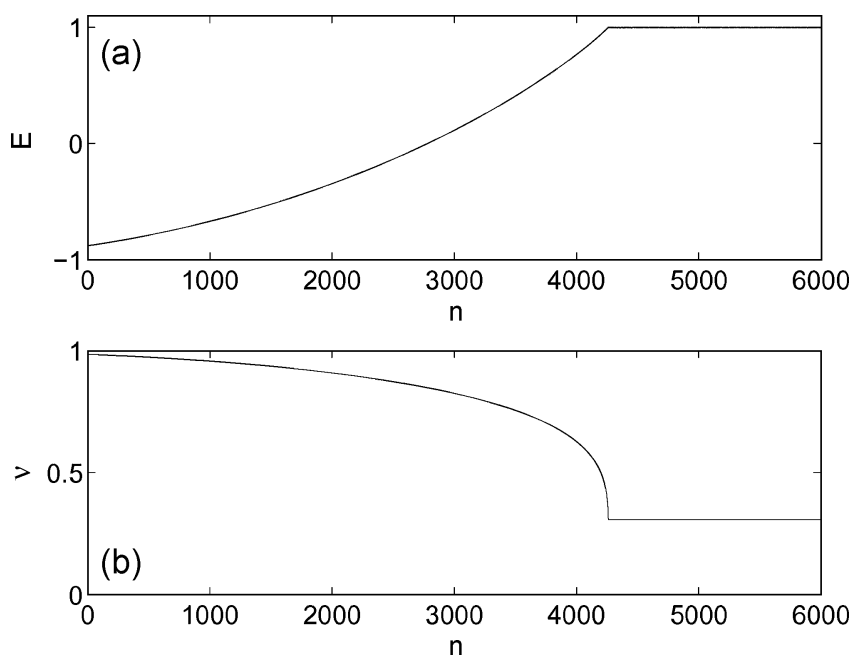

Fig. 2. (a) Energy of the oscillator as a function of oscillation period for $F=$ $10^{-4}$. The phase $\phi$ in (6) is chosen as in phase with $x(t)$ for $E<1.0$ and it remains constant for $E \approx 1.0$. Since $(6)$ represents a Hamiltonian system, the energy will oscillate about $E=1$ under the influence of external perturbation $F \sin (\nu t+\phi)$. (b) Frequency of the applied perturbation as a function of oscillation period.

Tuning the forcing frequency is not enough. We also need to control the phase. Since we want the energy to increase in time so that the system approaches the hyperbolic orbit, we have to adjust the phase $\phi$ so that the forcing term is always in phase with the system's oscillation. We do that by making adjustments in discrete times: every time $x$ crosses 0 in the positive direction, we change $\phi$ so that the forcing term is in phase with $x(t)$. For a real circuit, this could be achieved in continuous time by a phase-locking scheme. However, we note that the adjustment at each oscillation is small, because the oscillations depart only a little away from periodic motion. Therefore, the results would be essentially the same for both approaches. Imposing this phase-adjusting mechanism, we ensure that energy is always transferred from the perturbing force to the system, and not the opposite. If we do not do that, the phase would drift in time, and the energy would not increase monotonically in time, but would instead oscillate more or less randomly.

Now we apply the method described above to the system given by (6), with the potential $V$ given by (5). The system starts with the same initial conditions as in Fig. 1. The plot of energy versus time is shown in Fig. 2(a). We see that the method does indeed work: the energy increases monotonically with time, as the perturbation continuously injects energy into the system, until it reaches the threshold $E=E_{\max }$, at which chaos occurs. Fig. 2(b) shows the frequency variation corresponding to the changing energy, where it decreases as the energy increases and remains constant when the energy reaches unity. We find that $E$ will eventually reach $E_{\max }$ for any values of $F$, no matter how small (this is not true for dissipative systems, as we shall describe below). For perturbations of different magnitude, the difference is that the smaller the value of $F$, the longer it takes for $E$ to reach $E_{\max }$. For small enough $F$, we find that, approximately, this time is inversely proportional to $F$. Specifically, let $n_{\max }$ be the number of oscillations of the external excitations

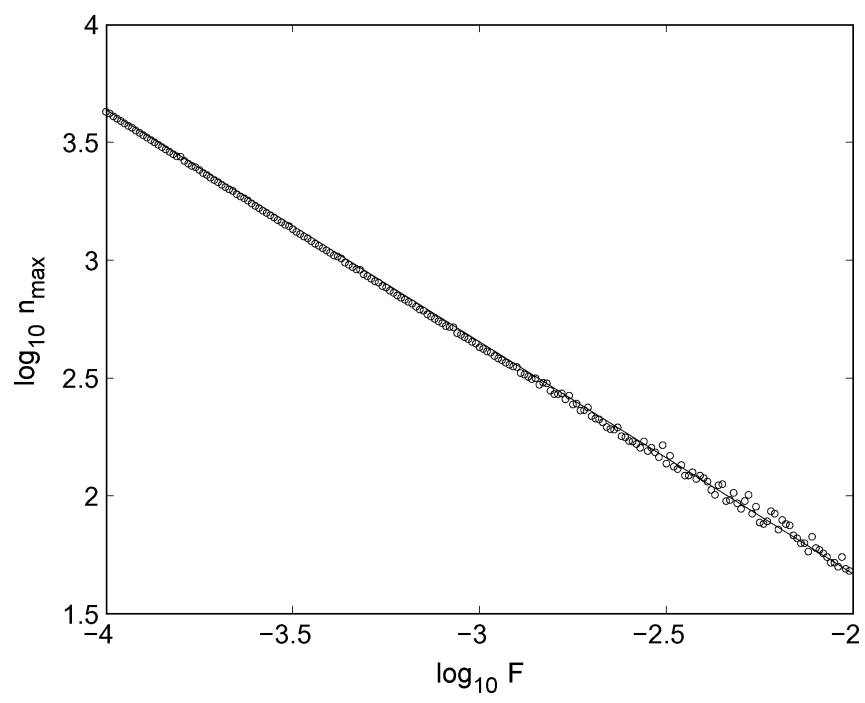

Fig. 3. Relation between $n_{\max }$, the number of oscillations of the external excitations required for energy $E$ to reach unity (i.e., for chaos to occur), and the perturbation strength $F$.

required for energy $E$ to reach unity. Fig. 3 shows the relationship between $n_{\max }$ and the perturbation strength $F$, where we observe the following:

$$
n_{\max } \sim F^{-1}
$$

This relation can be understood by writing down the energy function of the system: $E(t)=u^{2}(t) / 2+V(x)$, where $u(t)=$ $d x / d t$ is the velocity of the particle. Taking time derivative of $E(t)$, in combination with (6), gives

$$
\frac{d E}{d t}=u(t)\{F \sin [\nu(t) t+\phi(t)]\}
$$

Thus, we have

$$
1-E_{0}=\int_{E_{0}}^{1} d E=F \int_{0}^{t_{\max }} u(t) \sin [\nu(t) t+\phi(t)] d t .
$$

Under the resonant condition, the velocity $u(t)$ contains a term proportional to the driving and, hence, the integrand contains a term proportional to $\sin ^{2}[\nu(t) t+\phi(t)]$ which gives the major contribution proportional to $F t_{\max }$ (the integration of the remaining terms are approximately zero because of the long-time average of the sinusoidal functions). Since $t_{\max } \sim n_{\max }$, we have: $F n_{\max } \sim$ constant, which is the inverse relation (7).

After the energy reaches the threshold unity, the oscillatory behavior becomes chaotic. This is so because the system has been pushed to moving near the hyperbolic points about which there is typically a homoclinic or heteroclinic tangle between the stable and unstable manifolds of the fixed points and consequently chaos [31], [32]. Because there is no dissipation, the system energy remains to be $E=1$ (and the system therefore remains chaotic) even if the time variations in the frequency and phase of the perturbations are withdrawn. Fig. 4(a) shows the chaotic time series of $x$ (unwrapped with respect to $2 \pi$ ). 

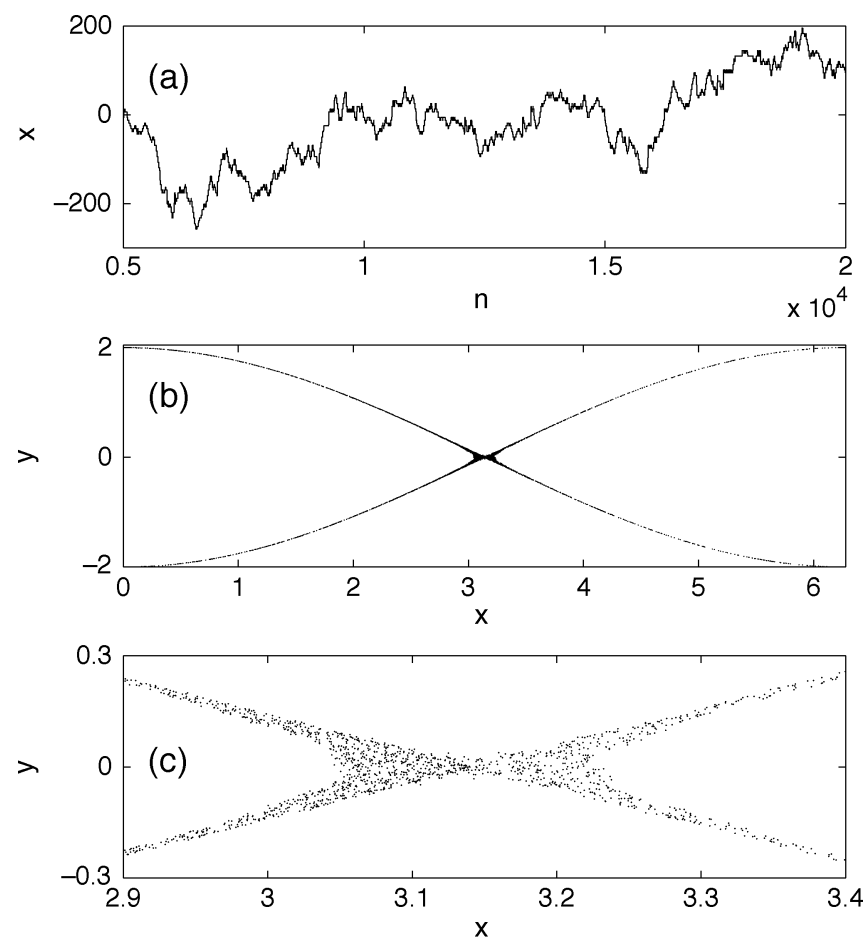

Fig. 4. For the nondissipative case: (a) induced chaotic time series $x_{n}$, (b) phase-space trajectory $(x, y)$ on the stroboscopic section defined by $t=2 \pi n / \nu(E=1)(n=1, \ldots)$, and (c) induced chaotic motion near the hyperbolic fixed point.

The phase-space trajectory $(x, y)$ on the stroboscopic section $t=2 \pi n / \nu(E=1)(n=1, \ldots)$ is shown in Fig. 4(b), where $0 \leq x \leq 2 \pi$. The induced chaotic behavior near the hyperbolic fixed points can be seen more clearly in Fig. 4(c).

We now address the important issue of dissipation. As we have seen, in the absence of dissipation, an arbitrarily small perturbation can drive the system to the vicinity of the hyperbolic orbit. We can expect that dissipation will change this. The reason becomes clear if we examine our method from the point of view of energy transfer. What the method does is to ensure that energy is always transferred from the external perturbation to the system. That is, we make sure that the external forcing always injects energy into the system (and never extracts energy from it). Without dissipation, the system's energy keeps increasing (albeit slowly), until the system inevitably reaches the energy of the hyperbolic orbit. In the presence of dissipation: although we still inject energy into the system using the external resonant perturbation, now energy is also getting lost. For a given energy, call the average energy input rate due to the forcing $\kappa_{\text {in }}$ and the energy output rate due to dissipation $\kappa_{\text {out }}$. Then, $\kappa_{\text {out }}$ usually increases the farther the system is from the equillibrium point, which could be a stable fixed point or a stable cycle. The total energy of the system will stop increasing when $\kappa_{\text {out }}$ equals $\kappa_{\text {in }}$. There are then two possible scenarios: 1) if this happens for an energy above the energy of the hyperbolic orbit $E_{\max }$, we will be able to achieve the goal of exciting the system to near $E_{\max }$ and therefore inducing chaos and 2) if, however, $\kappa_{\text {out }}$ becomes equal to $\kappa_{\text {in }}$ for energy $E$ less than $E_{\max }$, then the system will saturate at that energy, and we will not be able to push it to the neighborhood of the hyperbolic orbit. We can expect that, for a fixed forcing amplitude, as the dissipation increases from zero, a transition from case (1) to case (2) will occur.

To understand the effect of dissipation, we can add a term proportional to the velocity in our toy model

$$
\frac{d^{2} x}{d t^{2}}=-\frac{d V(x)}{d x}-\alpha \dot{x}+F \sin [\nu(t) t+\phi(t)]
$$

where $\alpha$ is the dissipation coefficient. The frequency and phase functions $\nu(t)$ and $\phi(t)$ of the perturbation are determined, as for the nondissipative case. For small enough dissipation, the system's oscillations are again nearly periodic, and all the assumptions we used for the calculation of $\phi$ and $\nu$ remain valid. One interesting feature in dissipative systems is that, when chaos is induced, in order to maintain it, the frequency of the external excitation needs to be adjusted continuously to keep the energy of the system at about 1 . The reason is that, when the system is driven to chaos, external energy is still needed to be delivered to the system to keep it in the chaotic state due to the dissipation. That is, it is still necessary to make the external perturbations resonant with the system. However, if continuous resonant perturbations are applied, it is likely that the system will settle into faster and faster rotational motion with monotonically increasing energy. Such a motion is in fact not chaotic. To avoid this situation, we monitor the dynamical variable $x(t)$ (in practice, this can be measured). If it exceeds $2 \pi$, we turn off the time variations in $\nu(t)$ and $\phi(t)$ so that the resonant condition is temporally not satisfied. As a result, little energy is transferred into the system so that its energy starts to decrease due to dissipation, at which point we turn on the time variations of $\nu(t)$ and $\phi(t)$ so that the condition of resonance is fulfilled again, and so on. This results in a continuous but small change in the frequency $\nu(t)$. This should be contrasted to the nondissipative case where, after chaos is induced, the system energy can be maintained at about $E=1.0$ even when the time variations in $\nu(t)$ and $\phi(t)$ are turned off [Fig. 2].

To confirm that chaos has indeed been induced, it is useful to compute the largest Lyapunov exponent $\lambda_{1}$, as the positiveness of the exponent is the defining characteristic of chaos. A complication is that the system equations under external time-dependent perturbation are not completely known. As a result, the standard procedure for computing the Lyapunov exponents in deterministic systems [33] is not applicable. Neither is the system random, so that the methods for computing Lyapunov exponents in random dynamical systems, such as those based on products of random matrices [34], [35], are not suitable either. However, the largest exponent $\lambda_{1}$ can be estimated by simply monitoring how the length of a small phase-space vector changes with time. In particular, we start with a small vector $\Delta \mathbf{x}\left(t_{0}\right)$ at $t_{0}=0$ and monitor its evolution up to time $t_{1}>t_{0}$, where $t_{1}$ is the time at which the vector $\Delta \mathbf{x}\left(t_{1}\right)$ is still small so that it can be related to $\Delta \mathbf{x}\left(t_{0}\right)$ approximately linearly. The ratio $r_{0}=\left|\Delta \mathbf{x}\left(t_{1}\right)\right| /\left|\Delta \mathbf{x}\left(t_{0}\right)\right|$ is then recorded and the vector $\Delta \mathbf{x}\left(t_{1}\right)$ is normalized so that it is small in length. The new vector is evolved in time, and the same procedure yields a new ratio $r_{1}$, and so on. The largest exponent $\lambda_{1}$ is approximately given by

$$
\lambda_{1} \approx \frac{1}{N} \sum_{i=0}^{N-1} \ln r_{i}, \quad \text { for } N \text { large. }
$$




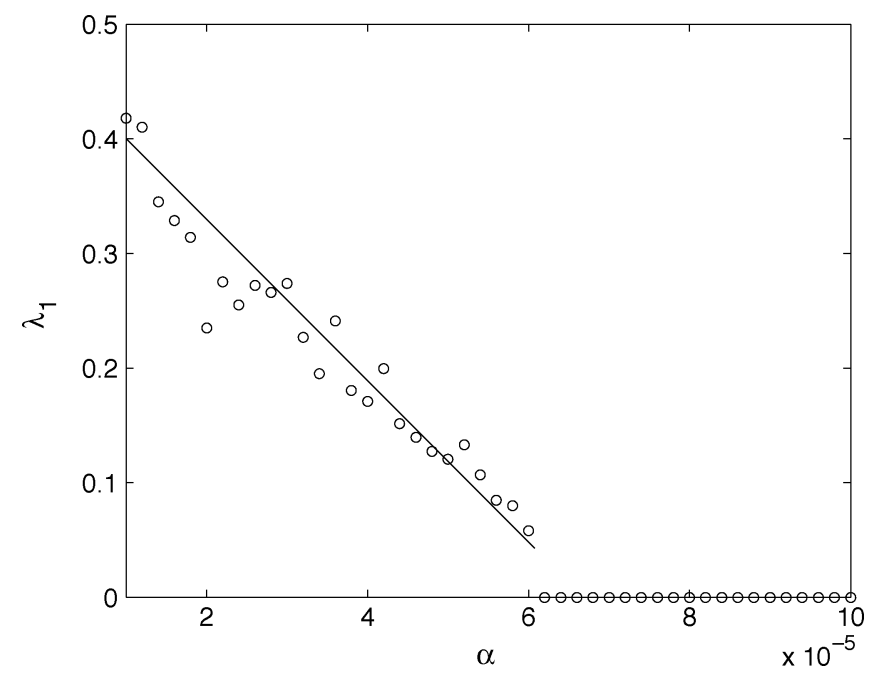

Fig. 5. For $F=10^{-4}$, the largest Lyapunov exponent $\lambda_{1}$ of the perturbed dynamics versus $\alpha$, where the exponent is positive for $\alpha<\alpha_{c}$. For each value of $\alpha$, the number of time steps is $N=(2 \pi / h) n$, where $h=0.01$ is the numerical integration step, and $n=10^{4}$ is the number of oscillatory cycles of the system.

Fig. 5(a) shows, for $F=10^{-4}$, the largest Lyapunov exponent versus $\alpha$, where we see that $\lambda_{1}>0$ for $\alpha<\alpha_{c}$, confirming that the dynamics of the system under external perturbation is indeed chaotic.

\section{EXPERIMENTAL INDUCTION OF CHAOS BY USING PLLS}

The method discussed above involves subjecting the oscillator to well designed periodic perturbations that can drive the system into higher and higher resonant states and eventually into chaos. We have argued theoretically and demonstrated numerically that it is possible to keep the oscillator in resonant states by using a perturbation with time-varying frequency and phase that match those of the oscillator, respectively, raising its energy until chaos is achieved. A convenient device that is capable of tracking the frequency and the phase of a nonlinear oscillator is PLLs [36]-[39].

To experimentally demonstrate the feasibility of our method, we construct a prototype circuit system consisting of a Duffingtype of circuit as the target oscillator to be driven into chaos and a PLL circuit that tracks the natural frequency and phase of the oscillator and delivers resonant perturbation. We will show in this Section that chaotic attractors can be readily induced in the Duffing oscillator that would otherwise exhibit a stable periodic attractor without the perturbation.

\section{A. The Duffing Oscillator and Circuit}

We have tested our method using the following periodically forced Duffing's oscillator (in a dimensionless form):

$$
\frac{d^{2} x}{d t^{2}}+\alpha \frac{d x}{d t}-x+x^{3}=A \sin \left(\nu_{1} t+\phi_{1}\right)
$$

where $\alpha=0.05, A=1, \nu_{1}=1$, and $\phi_{1}=0$. For this parameter setting the system exhibits a period-1 attractor and, hence,
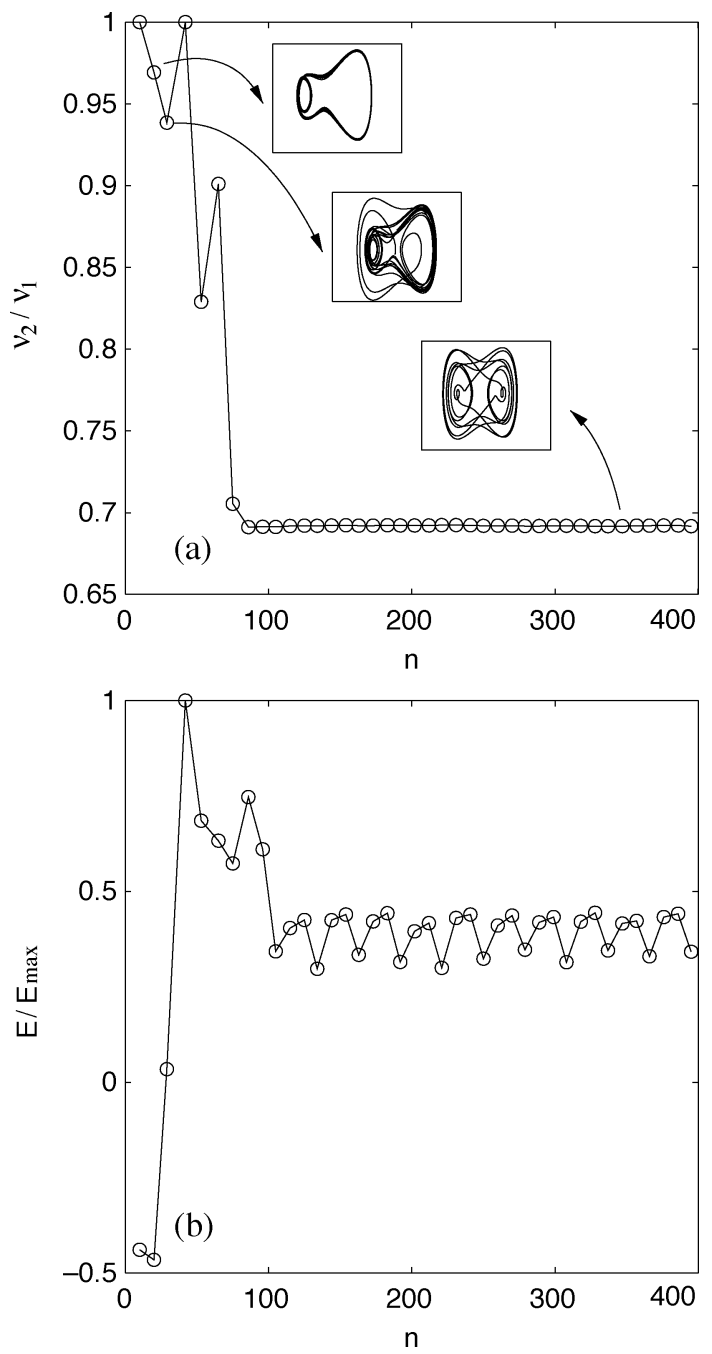

Fig. 6. For the periodically forced Duffing oscillator described by (9) that exhibits a period-1 attractor. (a) Change in the frequency in the external resonant perturbation and three representative attractors [in the $(x, d x / d t)$ plane] during the induction of chaos. (b) Evolution of the energy of the oscillator. Here, time $n$ denotes the number of cycles of the periodic forcing.

it can be regarded as being far from chaos. To induce chaos, we apply resonant perturbation of the form $F \sin \left[\nu_{2}(t) t+\phi_{2}(t)\right]$ to the right-hand side of (9), where $\nu_{2}(t)$ and $\phi_{2}(t)$ are estimated from the measured time series $x(t)$. Fig. 6(a) shows the evolution of the relative frequency $\nu_{2}(t) / \nu_{1}$ as chaos is being generated, together with a few representative phase-space plots of the attractor at different stages. We see that the frequency of the required resonant perturbation is decreased and remains at constant when chaos is induced, as predicted by our theory. Due to dissipation, the final frequency is finite. Fig. 6(b) shows the evolution of the energy of the system: $E(t)=(\dot{x})^{2} / 2+x^{2} / 2-$ $x^{4} / 4$, relative to its maximum value $E_{\max } \approx 0.37$. The initial energy assumes a negative value but it becomes approximately constant with small fluctuations after chaos sets in. Again, due to dissipation, the final average energy cannot reach its maximum possible value.

A circuit implementation of the Duffing's oscillator was proposed by Young and Silva [40], which uses square-wave driving 


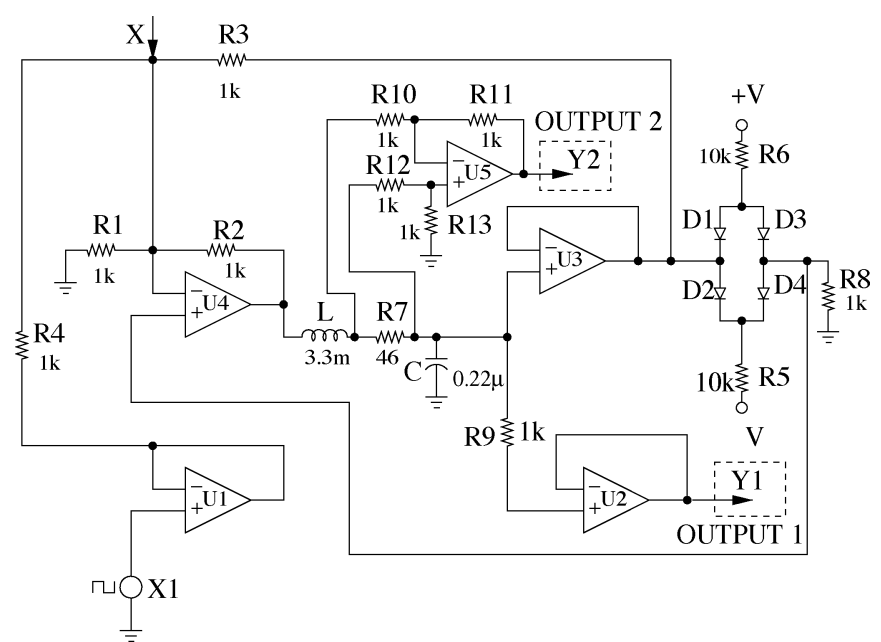

Fig. 7. Young-Silva circuit implementation of the Duffing oscillator. A differential amplifier (U5) was added to generate a single output proportional to the voltage across (R7) in order to visualize the phase plot of the voltage at $\mathrm{Y} 1$ versus that at $\mathrm{Y} 2$ on an oscilloscope.

and is capable of chaos-based information processing in a wide frequency range (kilohertz to gigahertz). For our experiment, we designed the Duffing circuit to be working in the range of 1-10 $\mathrm{kHz}$. To simplify the circuit, the nonlinear term $-x+x^{3}$ in (9) was replaced by a piecewise-linear function that can be implemented relatively easily. It was demonstrated that the resulting circuit preserves the essential dynamics of the original Duffing oscillator, including various periodic and chaotic behaviors [40].

Our circuit was built using the quad-operational-amplifier TL084 chip. The outputs are the voltages across the resistor R7 and from the operational-amplifier U2. The signals are digitally recorded using a digitizer (National Instrument) and analyzed using LabView (Version VI). In order to obtain the phase-space plot of the outputs for visualization, a differential amplifier using a TL082 chip was added to generate a voltage proportional to the potential across R7. The complete circuit diagram is shown in Fig. 7. The voltage $+\mathrm{V}$ and the supply voltages of the operational amplifiers are fixed as $9 \mathrm{~V}$. Utilizing the driving frequency as a bifurcation parameter, it was demonstrated that the Duffing circuit can generate a variety of nonlinear behaviors including periodic and chaotic attractors [40].

\section{B. $P L L$}

A PLL is a circuit that synchronizes a signal with another signal in both frequency and phase. We use PLL to generate a signal $V_{2}(t)$ that has the same frequency, and is synchronized in phase with an input signal $V_{1}(t)$. The input signal is from the Duffing's circuit with a periodic attractor, and the output signal is the resonant perturbation to be applied to the Duffing's circuit. Because of the match in both the frequencies and phases between the intrinsic oscillations of the Duffing's circuit and the external driving, the circuit can be brought into a hierarchy of resonant states, as suggested by our method. The basic block diagram of a PLL is shown in Fig. 8, which consists of a phase detector (PD), a loop filter with transfer function $G(s)$, and a voltage-controlled oscillator (VCO). The PD generates an output voltage $V_{p}(t)$ proportional to the phase error $\theta_{e}$ between

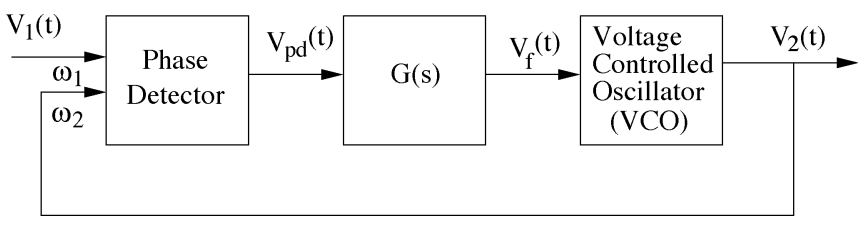

Fig. 8. Block diagram of a PLL. The output $V_{2}$ of the VCO (voltage controlled oscillator) is matched in frequency and synchronized in phase with the input $V_{1}$ to the PD.

the input signal $V_{1}(t)$ and the output $V_{2}(t)$ of the $\mathrm{VCO}$, as follows:

$$
V_{\mathrm{pd}}(t)=K_{\mathrm{pd}} \theta_{e}
$$

where $K_{\mathrm{pd}}$ is the PD gain. The alternating current components of the output of the PD are filtered out by the loop filter. The output of the VCO has a frequency that depends on the voltage input $V_{f}(t)$ to it and is given by

$$
\omega_{2}(t)=\omega_{0}+K_{v} V_{f}(t)
$$

where $\omega_{0}$ is the center frequency of the $\mathrm{VCO}$ and $K_{v}$ is the VCO gain.

A PLL works by matching the output frequency $\omega_{2}$ of the VCO with the input frequency $\omega_{1}$. The presence of the loop filter makes the PLL immune to noise, meaning that the PLL can track the input signal even if noise is present. The center frequency or free running frequency $\omega_{0}$ of the PLL is the frequency at which the VCO oscillates even if the input signal $V_{1}(t)$ is absent. The capture range $\Delta \omega_{C}$ of a PLL is the range of frequencies over which it can initially acquire a lock. Once a lock has been acquired, the PLL will continue tracking the input frequency over a range known as the lock range $\Delta \omega_{L}$. If the input frequency varies beyond the lock range, the locked loop will become unlocked. The capture range is always smaller than the lock range. The PLL can be designed appropriately such that its capture and the lock ranges are within the frequency range of the circuit of interest.

We emphasize that using a PLL causes the output signal to be synchronized in both frequency and phase, which is the essential requirement for driving the target system to chaos in our method. Note also that a PLL typically introduces a finite delay in the feedback loop which gives the system time to respond to the change in perturbation.

In our experiments, an LM565 PLL integrated circuit is used whose internal circuitry is shown in Fig. 9. The center frequency is given by

$$
\omega_{0}=\frac{0.6 \pi}{R_{1} C_{1}}
$$

and the lock range is

$$
\pm \Delta \omega_{L}= \pm \frac{8 \omega_{0}}{V_{c c}}
$$

where $V_{c c}$ is the supply voltage to the circuit. The outputs of the PLL at pins 4 and 5 are a square wave with the same frequency and phase as the input signal at pin 2. The output $Y_{1}$ of 


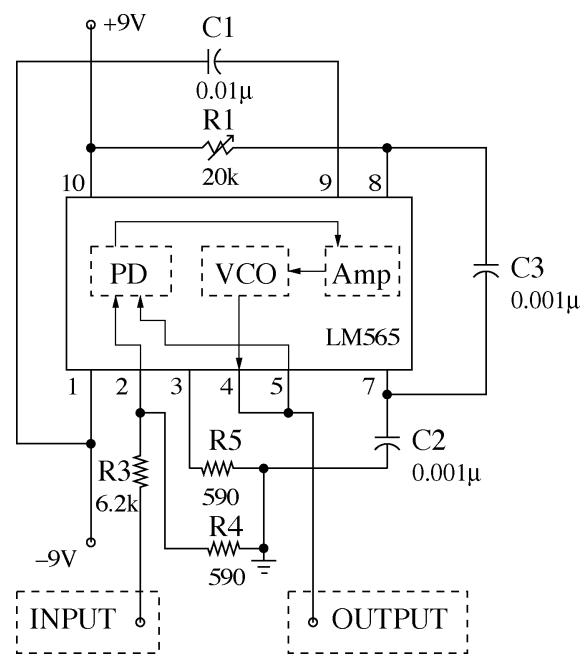

Fig. 9. Schematic diagram of the PLL circuit implemented using an LM565 chip in our experiments. The center frequency of the VCO is adjusted using the variable resistor $R_{1}$

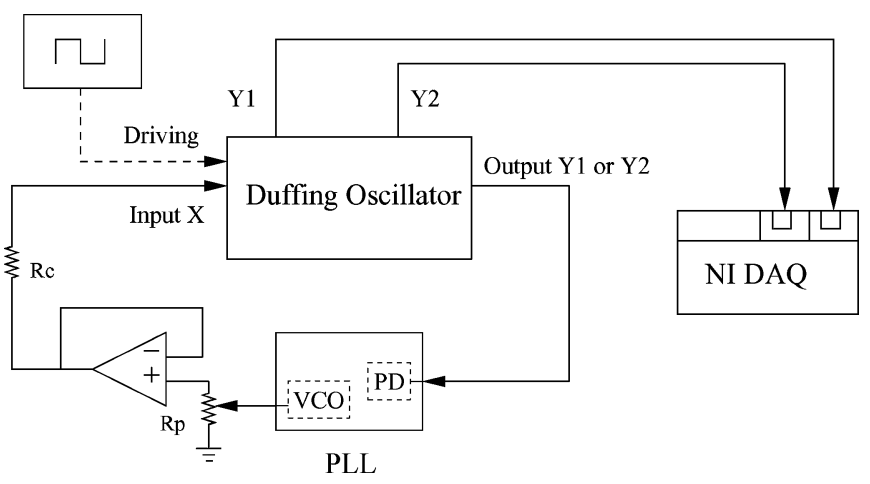

Fig. 10. Block diagram of our experimental scheme: the PLL is used to track the output frequency of the oscillator, and a signal at this frequency is used to drive the oscillator with the delay introduced by the PLL. A voltage divider circuit with a potentiometer is used to vary the output amplitude.

the Duffing circuit is fed to one of the inputs of the PD and the output of the VCO is used to feed the resonant perturbation to the Duffing circuit. A voltage divider at the output of the VCO is used to adjust the amplitude of the driving signal. Our experimental scheme can be summarized as the block diagram in Fig. 10 , where $R_{p}$ is a $10 \mathrm{k} \Omega$ potentiometer and $R_{c}$ is a $1-\mathrm{k} \Omega$ resistor.

\section{Experimental Results}

The Duffing circuit is driven using square wave signals. The output of the circuit can be periodic or chaotic, depending on the frequency selected and the amplitude of the square wave driving. Initially, we choose a frequency for which the Duffing circuit exhibits a stable periodic attractor. When the feedback loop is completed by using the output from the Duffing circuit as the input to the phase-looked loop, the circuit typically becomes chaotic, as desired.

To characterize the degree of the induced chaos in the circuit, we use the maximum Lyapunov exponent. Since we assume that the system equations are unknown, it is necessary to reconstruct the phase space based on a measured signal. We use the voltage signal $Y_{1}(t)$ from the port Y1 in the Duffing circuit and reconstruct from it a seven-dimensional dynamical system using the standard delay-coordinate embedding method [41], [42]. A straightforward strategy for estimating the maximum Lyapunov exponent is to directly monitor the growth of an infinitesimal vector in the reconstructed phase space [43]. In particular, let $\mathbf{x}_{n}$ be a state vector in the reconstructed phase space at time $n$ and $\mathbf{y}_{n}$ be its nearest-neighboring vector. Given a long time series $Y_{1}(t)$ and sampling time interval $t_{s}$, we assume that we have $N_{0}$ (large) iterations available for the phase-space vectors $\mathbf{x}_{n}$ and $\mathbf{y}_{n}$, where one iteration corresponds to one sampling interval. After $k$ iterations, the distance between the two vectors becomes $\left|\mathbf{y}_{n+k}-\mathbf{x}_{n+k}\right|$. We can then define the prediction error as

$$
p(k)=\frac{1}{N t_{s}} \sum_{n=1}^{N} \log _{2} \frac{\left|\mathbf{y}_{n+k}-\mathbf{x}_{n+k}\right|}{\left|\mathbf{y}_{n}-\mathbf{x}_{n}\right|}
$$

where $N_{0}$ iterations are used to search for the nearest neighbors, $N<N_{0}$, and $k<N_{0}$. If the dynamics is nonchaotic, the prediction error will fluctuate about a constant, except initially when the error increases rapidly from zero to the constant value. The slope of $p(k)$, which should fluctuate about zero, is an estimate of the maximum Lyapunov exponent. Usually, a histogram of the exponent can be constructed from independent runs and its center provides a reliable estimate for the exponent. For nonchaotic dynamics, the histogram should center at about zero. For chaotic dynamics, because of the exponential growth in the distance between nearby points, the prediction error $p(k)$ will increase with $k$ continuously until it becomes as large as the maximum variation of the original time series. The slope of $p(k)$ for $0<k \leq K_{0}$, where $K_{0}$ is the time when $p(k)$ becomes saturated, should be positive. Again, the center of the histogram of the maximum exponent provides a good estimate for it. In our computation we use $N_{0} \approx 50000, N=1000$, and $K=5000$. The number of runs used to construct the histogram of the maximum Lyapunov exponent is 1000. The time delay used in the delay-coordinate embedding is $\tau=50 t_{s}$, which corresponds to approximately half of the average oscillating period of the voltage signal.

To emphasize the point that chaotic attractor can be induced by our method when the system is far away from chaos, we show in Fig. 11 an experimentally obtained bifurcation diagram, where the parameter value indicated by the vertical arrow is used for experimental test. At this setting, the circuit exhibits a stable periodic attractor, as shown in Fig. 12(a) and (b), the phasespace plot from two outputs of the Duffing circuit and voltage signal $Y_{1}(t)$, respectively. The circuit is driven by a square wave of peak-to-peak amplitude of $4 \mathrm{~V}$ and frequency $4.5 \mathrm{kHz}$ to the port X1. Fig. 12(c) shows the evolution of the prediction error $p(k)$, which fluctuates around a constant (after a rapid initial increase). A histogram of the slope of $p(k)$ in the time after the initial transient increase is shown in Fig. 12(d). The center of the histogram is approximately zero, indicating that the maximum Lyapunov exponent is zero, as the case for a periodic attractor.

At this point, it is useful to explain what we mean when we say that the unperturbed system is far away from chaos. As shown in Fig. 11, because the attractor is period-1, it cannot be in a period window so that transient chaos associated with periodic 


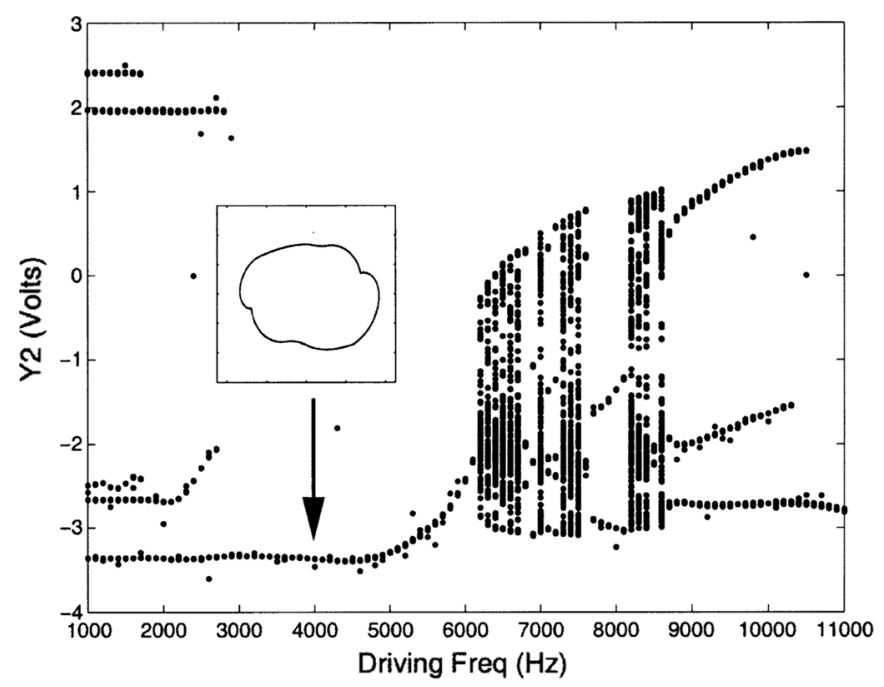

Fig. 11. Experimental bifurcation diagram for the Duffing circuit. The vertical arrow indicates the initial period-1 state of the system that is to be brought into chaos using a resonant perturbation.
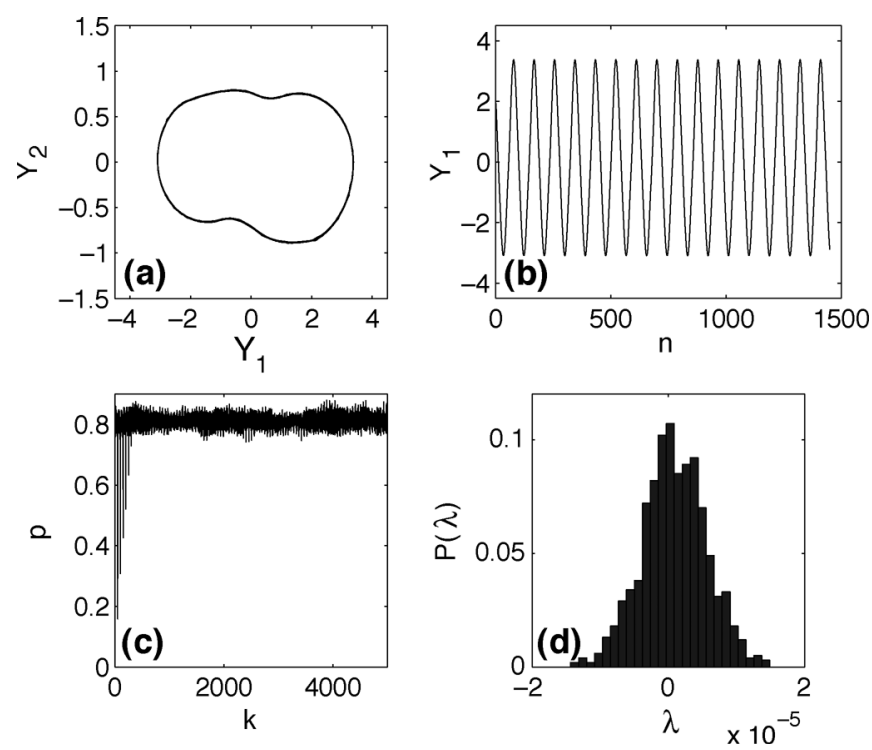

Fig. 12. (a) Phase-space plot of the attractor from the Duffing circuit in a stable periodic state. The quantities plotted are voltages from the ports Y1 and Y2 in the circuit (in volts). (b) Voltage signal $Y_{1}$, where $n$ is the iteration number. (c) Evolution of prediction error. (d) Histogram of the maximum Lyapunov exponent. The center of the histogram is approximately zero.

window is ruled out. On the other hand, careful examination of the measured time series indicated no sign of transient chaotic behavior associated with the period-1 attractor. Our case is thus quite different from that studied previously by In et al. [17] where the periodic state is embedded in a chaotic attractor. As a matter of fact, in many existing works based on chaos control [12]-[17], some sort of chaos, either a chaotic attractor or transient chaos as in a periodic window, was assumed. While in our case, as we described, there is no chaos initially, not even transient chaos. It is precisely in this sense that we say our system is far away from chaos.

Fig. 13(a) shows the phase-space plot of the Duffing circuit when resonant perturbations (the output of the PLL) of amplitude $1.3 \mathrm{~V}$ are applied to the point $\mathrm{X}$ of Fig. 7 through the resistor
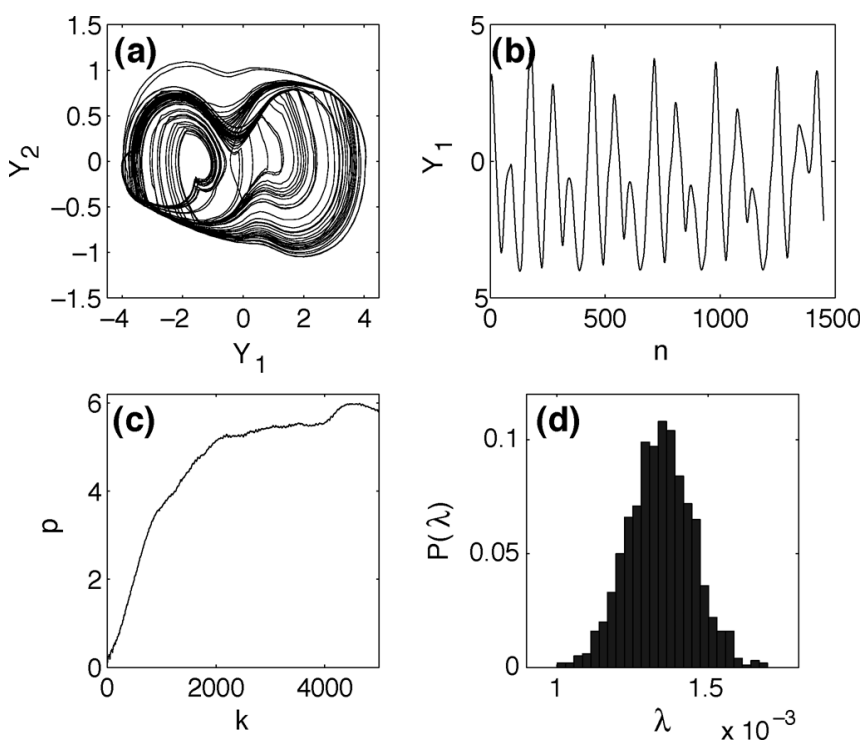

Fig. 13. (a) Phase-space plot of the attractor from the Duffing circuit under resonant driving. The quantities plotted are voltages from the ports Y1 and Y2 in the circuit (in volts). (b) Voltage signal $Y_{1}$, where $n$ is the iteration number. (c) Evolution of prediction error. (d) Histogram of the maximum Lyapunov exponent. The center of the histogram is positive, signifying chaos.
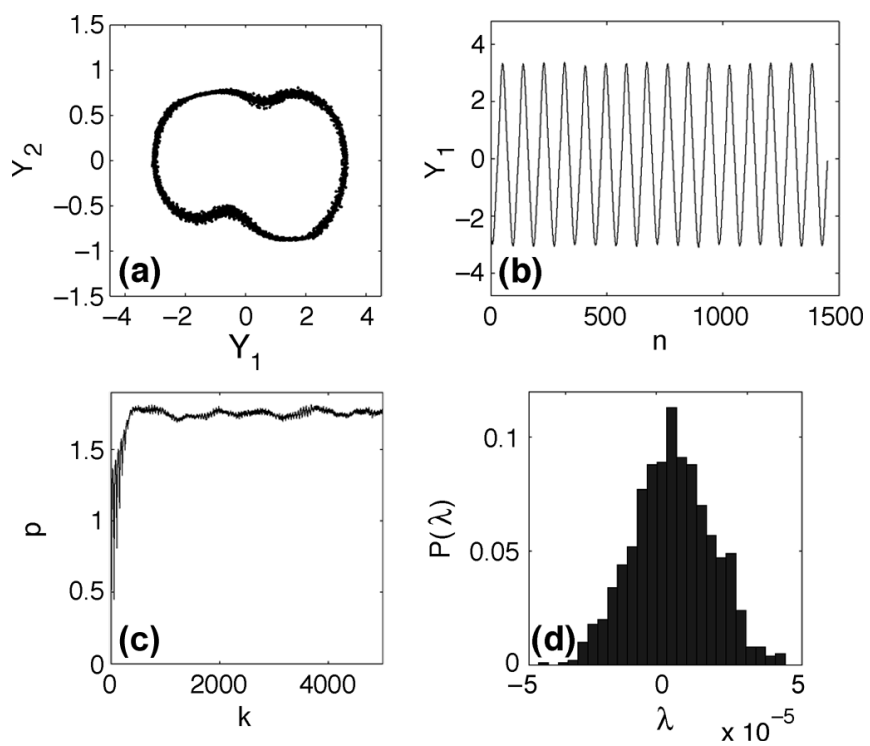

Fig. 14. (a) Phase-space plot of the attractor from the Duffing circuit in a stable periodic state under noise. (b) Voltage signal $Y_{1}$, where $n$ is the iteration number. (c) Evolution of prediction error. (d) Histogram of the maximum Lyapunov exponent. The center of the histogram is approximately zero, indicating lack of chaos.

$R_{c}$. The attractor is apparently chaotic, as can also be seen from the irregular voltage signal $Y_{1}(t)$ in Fig. 13(b). Fig. 13(c) shows the growth of the prediction error where it can be seen that the slope $\lambda_{1}$ is mostly positive. The histogram of $\lambda_{1}$ is observed to center at $\lambda_{1} \approx 1.3 \times 10^{-3}>0$, as shown in Fig. 13(d), which is clear evidence for chaos.

To show the effectiveness of the resonant driving in inducing chaos, we replace the resonant driving by a noisy signal of amplitude up to $6.4 \mathrm{~V}$ to the point $\mathrm{X}$ through a resistor $R_{c}$. The resulting phase-space plot and voltage signal are shown in Fig. 14(a) and (b), respectively. We see that the attractor, 
despite the large-amplitude noisy driving, appears to be only a smeared version of the original periodic attractor in Fig. 12(a). The evolution of the prediction error is shown in Fig. 14(c), which is similar to Fig. 12(c) in that it does not show a growth over time. The histogram of the maximum Lyapunov exponent is observed to center at $\lambda_{1} \approx 0$, as shown in Fig. 14(d), indicating lack of chaos.

\section{CONCLUSION}

We have developed a strategy, demonstrated by numerical computation and laboratory experiments, that a regular system far away from any complicated motion, can be driven to chaos through external time-dependent small perturbations. The key is resonant perturbation, whose frequency and phase match those of the nonlinear oscillator. We have shown that for experimental electronic circuit this can be conveniently implemented by using a PLL. We conceive the following situation of application: a signal is measured from a target system to be driven into chaos and the instantaneous frequency and phase of the system are computed using the signal, based on which continuous-time resonant perturbations are delivered to the system. As the frequency and the phase of the system are changing, those of the perturbations are changed accordingly to maintain the resonant condition. As a result of the continuous resonant excitations, the system can be driven to and maintained at a chaotic state. This can be achieved even if the amplitude of the perturbations is small (of course, the required amplitude depends on the amount of dissipation in the system). The external perturbations can be from a microwave source with time-varying frequency and phase.

Our strategy bears resemblance to the methodology of controlling chaos that has been pursued actively in the past following the idea of Ott, Grebogi, and Yorke [44], although the goals of inducing chaos and controlling chaos are exactly opposite. The basic observation in controlling chaos is that a chaotic set has typically embedded within itself an infinite number of unstable periodic orbits. Thus, one can choose an unstable periodic orbit that according to some criterion yields the best system performance, and apply small, judiciously chosen, temporal parameter perturbations to stabilize the system around this periodic orbit. To maintain the system at the desirable periodic motion, continuous perturbations are necessary [44], [45].

It should be noted that, in order to change the state of a circuit system, some parameters or dynamical variables must be accessible to perturbations. A basic assumption in our method is that no parameter or variable of the target system is accessible to $d i$ rect control, in contrast to the control-based methods [12]-[17]. As a result, only external excitation can be used to influence the system. The key difference between our method and the resonant-perturbation approach by Chacon [23] is that, in our case, we assume that the system equations are not available, versus Chacon's case where external controls are calculated based on full knowledge of the system equations. It is with respect to these points that we feel that our method of inducing chaos using resonant perturbations differs from and actually goes beyond the previous methods.

\section{ACKNOWLEDGMENT}

The authors thank M. Harrison for his insights and for suggesting the problem of inducing chaos in electronic circuits.
They are especially grateful to A. Young and C. P. Silva who generously provided them with the design of the Duffing circuit that made the experimental component of this paper possible. They are also grateful to A. P. S. de Moura for his help in the initial phase of the project.

\section{REFERENCES}

[1] J. P. Crutchfield and B. A. Huberman, "Fluctuations and the onset of chaos," Phys. Lett. A, vol. 77, pp. 407-410, 1980.

[2] J. P. Crutchfield, J. D. Farmer, and B. A. Huberman, "Fluctuations and simple chaotic dynamics," Phys. Rep., vol. 92, pp. 45-82, 1982.

[3] J. M. Deutsch, "Noise-induced phases of iterated functions," Phys. Rev. Lett., vol. 52, pp. 1230-1233, 1984.

[4] J. M. Deutsch, "The effect of noise on iterated maps," J. Phys. A-Math. Gen., vol. 18, pp. 1457-1474, 1985.

[5] J. Rössler, M. Kiwi, B. Hess, and M. Markus, "Modulated nonlinear processes and a novel mechanism to induce chaos," Phys. Rev. A, vol. 39, pp. 5954-5960, 1989.

[6] L. Yu, E. Ott, and Q. Chen, "Transition to chaos for random dynamical systems," Phys. Rev. Lett, vol. 65, pp. 2935-2938, 1990.

[7] L. Yu, E. Ott, and Q. Chen, "Fractal distribution of floaters on a fluid surface and the transition to chaos for random maps," Phys. D, vol. 53, pp. 102-124, 1991.

[8] J. B. Gao, S. K. Hwang, and J. M. Liu, "When can noise induce chaos?," Phys. Rev. Lett., vol. 82, pp. 1132-1135, 1999.

[9] S. K. Hwang, J. B. Gao, and J. M. Liu, "Noise-induced chaos in an optically injected semiconductor laser model," Phys. Rev. E, vol. 61, pp. 5162-5170, 2000.

[10] L. Billings and I. B. Schwartz, "Exciting chaos with noise: Unexpected dynamics in epidemic outbreaks," J. Math. Biol., vol. 44, pp. 31-48, 2002.

[11] Z. Liu, Y. C. Lai, L. Billings, and I. B. Schwartz, "Transition to chaos in continuous-time random dynamical systems," Phys. Rev. Lett., vol. 88 , no. 124101, 2002.

[12] Y. C. Lai and C. Grebogi, "Converting transient chaos into sustained chaos by feedback-control," Phys. Rev. E, vol. 49, pp. 1094-1098, 1994.

[13] S. J. Schiff, K. Jerger, D. H. Duong, T. Chang, M. L. Spano, and W. L. Ditto, "Controlling chaos in the brain," Nature, vol. 370, pp. 615-620, 1994.

[14] W. M. Yang, M. Z. Ding, A. J. Mandell, and E. Ott, "Preserving chaos: Control strategies to preserve complex dynamics with potential relevance to biological disorders," Phys. Rev. E, vol. 51, pp. 102-110, 1995.

[15] Y. Nagai and Y. C. Lai, "Selection of a desirable chaotic phase using small feedback-control," Phys. Rev. E, vol. 51, pp. 3842-3848, 1995.

[16] I. B. Schwartz and I. Triandaf, "Sustaining chaos by using basin boundary saddles," Phys. Rev. Lett., vol. 77, pp. 4740-4743, 1996.

[17] V. In, M. L. Spano, and M. Ding, "Maintaining chaos in high dimensions," Phys. Rev. Lett, vol. 80, pp. 700-703, 1998.

[18] R. Lima and M. Pettini, "Suppression of chaos by resonant parametric perturbations," Phys. Rev. A, vol. 41, no. 2, pp. 726-733, 1990.

[19] L. Fronzoni, M. Giocondo, and M. Pettini, "Experimental-evidence of suppression of chaos by resonant parametric perturbations," Phys. Rev. A, vol. 43, no. 12 , pp. 6483-6487, 1991.

[20] Y. Braiman and I. Goldhirsch, "Taming chaotic dynamics with weak periodic perturbations," Phys. Rev. Lett., vol. 66, no. 20, pp. 2545-2548, 1991.

[21] Z. Qu, G. Hu, G. Yang, and G. Qin, "Phase effect in taming nonautonomous chaos by weak harmonic perturbations," Phys. Rev. Lett., vol. 74, pp. 1736-1739, 1995.

[22] S. M. Booker, P. D. Smith, P. V. Brennan, and R. J. Bullock, "Designing input signals to disrupt commercial systems in band-A nonlinear dynamics approach," IEEE Trans. Circuits Syst. I, Fudam. Theory Appl. , vol. 49, no. 5, pp. 639-645, May 2002.

[23] R. Chacón, "Maintenance and suppression of chaos by weak harmonic perturbations: A unified view," Phys. Rev. Lett., vol. 86, pp. 1737-1740, 2001.

[24] Y. C. Lai, U. Feudel, and C. Grebogi, "Scaling behavior of transition to chaos in quasi-periodically driven systems," Phys. Rev. E, vol. 54, pp. 6070-6073, 1996.

[25] M. A. Harrison and Y. C. Lai, "A route to high-dimensional chaos," Phys. Rev. E, vol. 59, pp. R3799-R3802, 1999.

[26] M. A. Harrison and Y. C. Lai, "Bifurcation to high-dimensional chaos," Int. J. Bifur. Chaos, vol. 10, pp. 1471-1483, 2000. 
[27] R. L. Davidchack and Y. C. Lai, "Characterization of transition to chaos with multiple positive-Lyapunov exponents by unstable periodic orbits," Phys. Lett. A, vol. 270, pp. 308-313, 2000.

[28] V. In, S. E. Mahan, W. L. Ditto, and M. L. Spano, "Experimental maintenance of chaos," Phys. Rev. Lett., vol. 74, pp. 4420-4423, 1995.

[29] S. Banerjee, J. A. Yorke, and C. Grebogi, "Robust chaos," Phys. Rev. Lett., vol. 80, pp. 3049-3052, 1998.

[30] Y.-C. Lai, A. Kandangath, S. Krishnamoorthy, J. A. Gaudt, and A. P. S. de Moura, "Inducing chaos by resonant perturbations: Theory and experiment," Phys. Rev. Lett., vol. 94, p. 214101, 2005.

[31] A. J. Lichtenberg and M. J. Lieberman, Regular and Stochastic Motion. New York: Springer-Verlag, 1983.

[32] L. E. Reichl, The Transition to Chaos in Conservative Classical Systems: Quantum Manifestations. New York: Springer-Verlag, 1992.

[33] J. P. Eckmann and D. Ruelle, "Ergodic theory of chaos and strange attractors," Rev. Mod. Phys., vol. 57, pp. 617-656, 1985.

[34] J. Cohen, H. Kesten, and C. M. Newman, Eds., Random Matrices and Their Applications. Providence, RI: American Mathematical Society, 1984.

[35] J. M. Deutsch and G. Paladin, "Product of random matrices in a microcanonical ensemble," Phys. Rev. Lett, vol. 62, pp. 695-699, 1989.

[36] P. Horowitz and W. Hill, The Art of Electronics. New York: Cambridge Univ. Press, 1989.

[37] P. V. Brennan, Phase-Locked Loops: Principles and Practice, 1st ed. London: McGraw-Hill, 1996.

[38] E. Bradley, "Using chaos to broaden the capture range of a phaselocked loop," IEEE Trans. Circuits Syst. I, Fudam. Theory Appl., vol. 40, no. 8, pp. 808-818, Aug. 1993.

[39] E. Bradley and D. E. Straub, "Using chaos to broaden the capture range of a phase-locked loop: Experimental verification," IEEE Trans. Circuits Syst. I, Fudam. Theory Appl. , vol. 43, no. 9, pp. 914-922, Sep. 1996.

[40] C. P. Silva and A. M. Young, "Introduction to chaos based communication and signal processing," in Proc, IEEE Aerosp. Conf., 2000, vol. 1, pp. 279-299.

[41] F. Takens, "Detecting strange attractors in fluid turbulence," in Dynamical Systems and Turbulence, D. Rand and L. S. Young, Eds. Berlin, Germany: Springer-Verlag, 1981, pp. 366-381.

[42] H. Kantz and T. Schreiber, Nonlinear Time Series Analysis, 1st ed. Cambridge, U.K.: Cambridge Univ. Press, 1997.

[43] A. Wolf, J. B. Swift, L. Swinney, and J. A. Vastano, "Determining Lyapunov exponents from a time series," Phys. D, vol. 16, pp. 285-317, 1985.

[44] E. Ott, C. Grebogi, and J. A. Yorke, "Controlling chaos," Phys. Rev. Lett, vol. 64, pp. 1196-1199, 1990.

[45] S. Boccaletti, C. Grebogi, Y. C. Lai, H. Mancini, and D. Maza, "The control of chaos: Theory and applications," Phys. Rep., vol. 329, pp. 103-197, 2000.

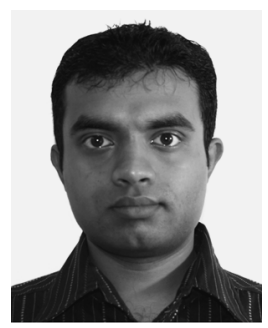

Anil Kandangath is currently working toward the M.S. degree in electrical engineering at Arizona State University, Tempe, with an emphasis on signal processing and telecommunications.

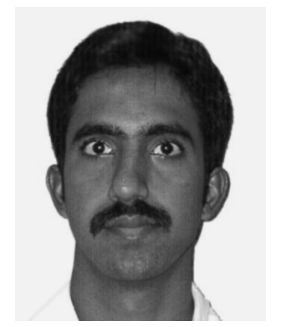

Satish Krishnamoorthy is currently working toward the M.S. degree in electrical engineering at Arizona State University, Tempe, with an emphasis on VLSI circuits and systems.

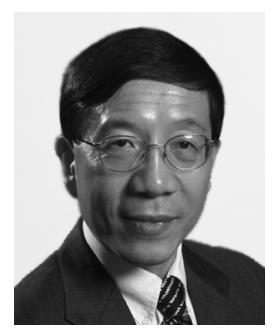

Ying-Cheng Lai received the Ph.D. degree from University of Maryland, College Park, in 1992.

He has been a Professor of Electrical Engineering at Arizona State University, Tempe, since 2001, and has been a Fellow of the American Physical Society since 1999. His research interests are nonlinear dynamics, signal processing, complex networks, and computational biology.

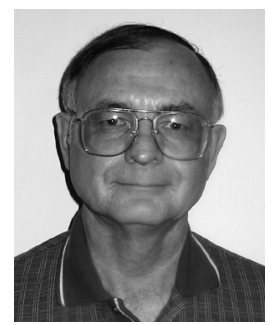

John A. Gaudet (M'93) received the B. A. degree from the College of the Holy Cross, Worcester, MA, in 1969, the M.S. degree from the University of Notre Dame, Notre Dame, IN, in 1971, and the Ph.D. from the Air Force Institute of Technology, Wright-Patterson, $\mathrm{OH}$, in 1981.

During a 22-year U.S. Air Force career, he conducted research on numerical modeling of electromagnetic pulses, developed space experiments for plasma satellite interactions, and directed pulsed power and high power microwave (HPM) research. He also taught physics at the Air Force Academy. From 1993 until 2001, he was a staff member at the New Mexico Engineering Research Institute working on both narrowband and ultra-wide band high-power microwave projects for the Air Force Research Laboratory (AFRL). In 2001, he joined the Electrical and Computer Engineering Department at The University of New Mexico (UNM), Kirtland AFB, as Associate Research Professor. He continues to work with AFRL, concentrating on nonlinear effects in circuits caused by RF interference. At UNM, he studies the electrical breakdown characteristics of advanced materials for use in compact pulsed power applications.

Dr. Gaudet is a charter member of the Directed Energy Professional Society. 